\title{
Apoio social e resiliência: um olhar sobre a maternidade na adolescência
}

\author{
Social support and resilience: a look at adolescent motherhood \\ Apoyo social y resiliencia: una mirada sobre la maternidad en la adolescencia \\ Bianca Gansauskas de Andrade ${ }^{1}$ (1) https://orcid.org/0000-0002-3822-9770 \\ Cláudia Alves de Assis ${ }^{1}$ @ https://orcid.orgy0000-0002-8765-5098 \\ Débora Cristina de Melo Lima' ic https://orcid.org/000-0001-6021-7111 \\ Lucila Faleiros Neves ${ }^{1}$ @i https://orcid.org/0000-0003-0162-6614 \\ Leticia Aparecida da Silva ${ }^{1}$ ic https://orcid.org/0000-0003-4831-6525 \\ Reginalice Cera da Silva ${ }^{1}$ ie https://orcid.org/0000-0002-3705-8447 \\ Lislaine Aparecida Fracolli ${ }^{1}$ io https://orcid.org/0000-0002-0936-4877 \\ Anna Maria Chiesa ${ }^{1}$ io hitps://orcid.org/0000-0002-8343-3922
}

Como citar:

Silva BC, Silva RC, et al. Apoio social e resiliência: um olhar sobre a maternidade na adolescência. Acta Paul Enferm. 2022;35:APE03341.

DOI

http://dx.doi.org/10.37689/acta-ape/2022A003341

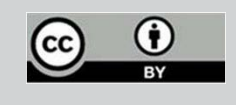

Descritores

Resiliência psicológica; Apoio social; Gravidez na adolescência; Promoção da saúde

Keywords

Resilience, psychological; Social support; Pregnancy in adolescence; Health promotion

Descriptores

Resiliencia psicológica; Apoyo social; Embarazo en adolescência; Promoción de la salud

Submetido 20 de Dezembro de 2019

Aceito

26 de Maio de 202

Autor correspondente

Bianca Gansauskas de Andrade

E-mail: bgansauskas@gmail.com

Editor Associado (Avaliação pelos pares): Denise Myuki Kusahara

(https://orcid.org/0000-0002-9498-0868) Escola Paulista de Enfermagem, Universidade Federal de São Paulo, São Paulo, SP, Brasil

\section{Resumo}

Objetivo: Verificar a influência do apoio social no processo de resiliência de mães adolescentes.

Métodos: Estudo exploratório, descritivo, transversal, de abordagem quantitativa. Foram sujeitos da pesquisa 48 adolescentes atendidas em Unidades Básicas de Saúde, no estado de São Paulo, em 2016. Os dados foram coletados dos seguintes instrumentos: a) questionário sociodemográfico; b) Escala de Resiliência desenvolvida por Wagnild e Young (1993), adaptada por Pesce et al. (2005); c) Escala de Apoio Social utilizada no Medical Outcomes Study , adaptada por Griep et al. (2005).

Resultados: Os resultados mostraram alta pontuação nas Escalas de Resiliência e de Apoio Social das adolescentes. Destas 70,83\% apresentaram atraso escolar significativo e 75\% não trabalhavam. Foram encontradas associações significativas entre os fatores da Escala de Resiliência e as dimensões da Escala de Apoio Social. Foi identificada uma correlação inversa entre o fator "Independência e determinação", da Escala de Resiliência e a dimensão "Afetiva" da Escala de Apoio Social. Portanto, quanto maior o "apoio afetivo" percebido pelas mães adolescentes, menor a "independência e determinação" apresentada por elas.

Conclusão: 0 estudo aprofunda os conhecimentos sobre a influência do apoio social no processo de resiliência de mães adolescentes. Evidenciaram-se questões de gênero e a importância de políticas intersetoriais com foco em mães e pais adolescentes que fortaleçam o apoio social.

\section{Abstract}

Objective: To verify the influence of social support on the resilience process of adolescent mothers.

Methods: Exploratory, descriptive, cross-sectional study with a quantitative approach. There were 48 adolescents research subjects, who attended Primary Care Centres, in the state of São Paulo, in 2016. Data were collected from the following instruments: a) sociodemographic questionnaire; b) Resilience Scale developed by Wagnild and Young (1993), adapted by Pesce et al. (2005); c) Social Support Scale used in the Medical Outcomes Study, adapted by Griep et al. (2005).

Results: The results showed high scores on the Resilience and Social Support Scales of the adolescents. Of these, $70.83 \%$ had significant school delays and $75 \%$ did not work. Significant associations were found between the factors of the Resilience Scale and the dimensions of the Social Support Scale. An inverse correlation was identified between the "Independence and determination" factor of the Resilience Scale and the "Affective" dimension of the Social Support Scale. Therefore, the greater the "affective support" perceived by teenage mothers, the less "independence and determination" they present.

Conclusion: The study deepens the knowledge about the influence of social support on the resilience process of teenage mothers. Gender issues and the importance of intersectoral policies focused on teenage mothers and fathers that strengthen social support were highlighted. 


\section{Resumen}

Objetivo: Verificar la influencia del apoyo social en el proceso de resiliencia de madres adolescentes.

Métodos: Estudio exploratorio, descriptivo, transversal, de enfoque cuantitativo. Los sujetos de la investigación fueron 48 adolescentes atendidas en Unidades Básicas de Salud, en el estado de São Paulo, en 2016. Los datos fueron recopilados a través de los siguientes instrumentos: a) cuestionario sociodemográfico; b) Escala de Resiliencia, elaborada por Wagnild y Young (1993), adaptada por Pesce et al. (2005); c) Escala de Apoyo Social utilizada en el Medical Outcomes Study, adaptada por Griep et al. (2005).

Resultados: Los resultados reflejaron una puntuación elevada en la Escala de Resiliencia y de Apoyo Social de las adolescentes. De ellas, el 70,83 \% presentó retraso escolar significativo y el $75 \%$ no trabajaba. Se descubrieron relaciones significativas entre los factores de la Escala de Resiliencia y las dimensiones de la Escala de Apoyo Social. Se identificó una correlación inversa entre el factor "independencia y determinación" de la Escala de Resiliencia y la dimensión "afectiva" de la Escala de Apoyo Social. Por lo tanto, cuanto mayor era el "apoyo afectivo" percibido por las madres adolescentes, menor "independencia y determinación" presentaron.

Conclusión: El estudio profundiza los conocimientos sobre la influencia del apoyo social en el proceso de resiliencia de madres adolescentes. Se evidenciaron cuestiones de género y la importancia de políticas intersectoriales con foco en madres y padres adolescentes que fortalezcan el apoyo social.

\section{Introdução}

A maternidade na adolescência é considerada uma situação de vulnerabilidade com impactos profundos na vida das adolescentes e na saúde do bebê. ${ }^{(1,2)} \mathrm{A}$ Organização Mundial de Saúde considera adolescente a pessoa entre 10 e 19 anos. ${ }^{(3)}$ A vulnerabilidade considera os aspectos sociais e de acesso aos serviços de saúde além dos fenômenos individuais e biológicos ao reconhecer as necessidades de saúde. ${ }^{(4,5)}$ Estudos apontam uma assistência de pré-natal e parto deficientes para gestantes adolescentes, contribuindo para maior incidência de prematuridade e baixo peso ao nascer. ${ }^{(6,7)}$ A baixa escolaridade e a consequente entrada precoce e precarizada no mercado de trabalho, somadas à pobreza, tornam a gravidez na adolescência um dos mais importantes fatores para a perpetuação de ciclos intergeracionais de pobreza e exclusão. ${ }^{(1,2,8)}$

$\mathrm{Na}$ ausência de medidas de mitigação das consequências negativas da gestação e maternidade na adolescência, como no caso de gestação indesejada e falta de apoio familiar e social, esta pode se constituir em adversidade por representar um impedimento para avanços no status educacional e socioeconômico das mulheres em todas as partes do mundo. ${ }^{(9)}$ Corroborando, pesquisas realizadas com mães adolescentes de baixa renda mostram que as dificuldades de acesso ao trabalho e ao bem-estar socioeconômico, foram os principais riscos sociais enfrentados por elas. ${ }^{(2,10)}$ Contrariando estas afirmaçôes, alguns estudos indicam que as precárias condições socioeconômicas e as oportunidades restritas precedem a gravidez na adolescência. ${ }^{(1-13)}$ Portanto, esse fenômeno é, simultaneamente, causa e conse- quência das violaçóes de direitos, minando a capacidade de uma adolescente de exercer seus direitos à educação, saúde e autonomia. ${ }^{(9)}$

$\mathrm{O}$ apoio social é um dos fatores protetores que auxiliam todas as mulheres no processo de gestação e maternagem, sobretudo entre as mães adolescentes na construção da resiliência. ${ }^{(9)}$

Apoio social refere-se aos recursos disponibilizados por outras pessoas a alguém que se encontra em situação de necessidade e pode corresponder a diferentes funçôes, como apoio emocional, material ou afetivo. ${ }^{(14-16)} \mathrm{O}$ apoio social pode ser um elemento a favorecer o empowerment, ${ }^{(17)}$ pois contribui no sentido de criar uma sensação de coerência e controle da vida ${ }^{(18)}$ o que beneficiaria o estado de saúde das pessoas.

Resiliência se refere à capacidade que o ser humano tem de se recuperar psicologicamente quando é submetido a adversidades, violências e catástrofes na vida; corresponde à capacidade de pessoas, grupos e/ou comunidades não só de resistir às adversidades, mas utilizá-las em seus processos de desenvolvimento pessoal e crescimento social. ${ }^{(19)}$

Considerando o panorama de vulnerabilidade acerca da maternidade na adolescência foram levantados os seguintes questionamentos: 1) como se caracterizam a resiliência e o apoio social de mães adolescentes que vivem em contextos vulneráveis? 2) existe relação da resiliência e do apoio social com as características sociodemográficas? e 3) existe correlação significativa entre apoio social e resiliência?

O objetivo deste estudo foi verificar a influência do apoio social no processo de resiliência de mães adolescentes. 


\section{Métodos}

Trata-se de um estudo exploratório descritivo, transversal de abordagem quantitativa. A população foi de 48 mães adolescentes de 10 a 19 anos, com filhos de até um ano de idade, atendidas em cinco UBS, no estado de São Paulo, no ano de 2016. Os critérios de inclusão foram: a idade entre $10 \mathrm{a}$ 19 anos; ser mãe adolescente; ter algum dos filhos com até um ano de idade e possuir condiçóes de se comunicar verbalmente. Os critérios de exclusão foram: náo apresentar endereço ou forma de localização correta e impossibilidade de localização após a terceira tentativa de contato presencial.

O cálculo da amostra aleatória simples considerou o nível de confiança de $95 \%$, o poder de teste de $95 \%$ e o valor correlação entre a resiliência e o apoio social de 0,3 . Utilizou-se para o cálculo da amostra o software $G^{*}$ Power 3.1.9.2, elaborado por Faul, ${ }^{(20)}$ resultando em uma amostra de 138 e, após, foi feita uma correção para populaçáo, utilizando a seguinte fórmula:

$$
\mathrm{n}^{*}=\frac{\mathrm{n} \mathrm{x} \mathrm{N}}{\mathrm{n}+\mathrm{N}-1}=47
$$

$\mathrm{n}=138$ (número da amostra sem a correção para população finita)

$\mathrm{N}=70$ (número estimado da população total)

Com a correção para amostra finita, chegou-se à amostra de 47 adolescentes, no entanto foi possível entrevistar 48 delas.

A coleta de dados foi realizada entre os meses de julho e agosto de 2016. Nesta pesquisa foram aplicados os seguintes instrumentos: questionário sociodemográfico, Escala de Resiliência $(\mathrm{ER})^{(21)}$ e Escala de Apoio Social utilizada no Medical Outcomes Study (MOS) [EAS (MOS)].(16) O questionário sociodemográfico foi elaborado pela própria pesquisadora e possui questóes relacionadas às seguintes variáveis: idade; número de filhos; escolaridade; estado civil; atividade econômica e tempo que a adolescente passa com seu (s) filho(s). Em relação à escolaridade, foi considerada a última série concluída com aprovação ou a que a adolescente estava cursando no momento da entrevista, sem ser questionado se o abandono escolar ocorreu antes ou depois da gestação. A ER foi desenvolvida por Wagnild e Young, ${ }^{(22)}$ adaptada para o português por Pesce ${ }^{(21)}$ em uma população de adolescentes. Essa escala é utilizada para medir níveis de adaptação psicossocial positiva em face de eventos de vida importantes. Possui 25 itens descritos de forma positiva com resposta tipo Likert variando de 1 (discordo totalmente) a 7 (concordo totalmente). ${ }^{(21)} \mathrm{O}$ apoio social foi mensurado pela EAS (MOS), adaptada para o português por Griep et al. ${ }^{(16)}$ Esse instrumento foi concebido para abranger cinco dimensóes de apoio social: material; afetiva; interaçáo social positiva; emocional. Nesta escala as maiores pontuaçōes indicam apoio social satisfatório. ${ }^{(15)}$

Os dados foram organizados e analisados utilizando o software Statistical Package for the Social Sciences (SPSS) 22,0. O teste de Shapiro-Willk foi utilizado para verificar se as amostras obedeciam a distribuição normal e, como a maior parte das variáveis não seguia o padrão normal, foi realizada a correlação de postos de Spearman para verificar a correlação entre as variáveis. O nível de significância considerado foi $\mathrm{p}<0,05$.

Esta pesquisa foi realizada em conformidade com a Resolução no 466/2012 do Conselho Nacional de Saúde, apreciada pelo Comitê de Ética da Escola de Enfermagem da Universidade de São Paulo de número 1.622.591, CAAE: 55713216.6.0000.5392 e pela Secretaria Municipal de Saúde da Prefeitura de Bertioga que deu a sua anuência. ${ }^{(23)}$

\section{Resultados}

\section{Caracterização da amostra}

A população da pesquisa foi composta por 48 mães adolescentes.-

A idade média das mães foi de 17,58 anos, com desvio padrão de $1,39 \mathrm{e}$, as adolescentes com idade entre 18 e 19 anos representam, 60,42\% ( $n=29)$ da amostra. $68,74 \%(\mathrm{n}=33)$ das adolescentes estavam em uma uniáo estável ou casadas e $75 \%(n=36)$ não estavam trabalhando. $\mathrm{O}$ número de filhos por adolescente neste estudo foi de 1,15 filhos com um desvio padrão de mais ou menos 0,35 .

Em relação à escolaridade, verificou-se que $75 \%$ $(\mathrm{n}=36)$ das adolescentes pararam de estudar; $25 \%$ 
$(n=12)$ continuaram estudando e 70,83\% ( $n=34)$ encontravam-se com atraso escolar.

A média do nível de escolaridade foi de 9,31 anos com um desvio padrão de 2,08. Das entrevistadas, $14,58 \%(\mathrm{n}=7)$ completaram o $3^{\circ}$ ano do ensino médio. Em relação ao ensino fundamental, 12, $50 \%(n=6)$ cursaram até o $9^{\circ}$ ano; $10,42 \%(n=5)$ o $8^{\circ}$ ano; $12,50 \%(n=6)$ o $7^{\circ}$ ano; $2,08 \%(n=1)$ o $5^{\circ}$ ano e $2,08 \%(n=1)$ o $4^{\circ}$ ano.

Nesta pesquisa, observou-se uma correlação inversa entre união estável/casamento com continuidade dos estudos, ou seja, à medida que aumenta o número de mães adolescentes em união estável/casamento, diminui o número destas que continuam a estudar (rho $=-0,332 ; p=0,023)$.

\section{Caracterização da resiliência}

Os resultados da resiliência das mães adolescentes foram obtidos por meio da Escala de Resiliência e definidos de acordo com a classificação de resiliência da autora, Wagnild. Das pesquisadas, 8,33\% $(n=4)$ apresentaram muito alta resiliência, 35,42\% $(\mathrm{n}=17)$ alta resiliência, $31,25 \%(\mathrm{n}=15)$ moderada-alta resiliência, 20,83\% ( $\mathrm{n}=10)$ moderada-baixa resiliência, 2,08\% ( $\mathrm{n}=1)$ baixa resiliência, e 2,08\% ( $\mathrm{n}=1)$ muito baixa resiliência. Observa-se que $75 \%$ delas atingiram pontuaçóes entre moderada-alta resiliência e muito alta resiliência (Tabela 1 ).

Tabela 1. Caracterização da resiliência das mães adolescentes e dos fatores que compõem a Escala de Resiliência

\begin{tabular}{|c|c|c|c|c|c|}
\hline Fatores & $\mathrm{n}$ & $\begin{array}{l}\text { Mínimo } \\
\text { do } \\
\text { estudo }\end{array}$ & $\begin{array}{c}\text { Máximo } \\
\text { do } \\
\text { estudo }\end{array}$ & Média & $\begin{array}{l}\text { Desvio- } \\
\text { padrão }\end{array}$ \\
\hline 1. Resolução de ações e valores & 48 & 2,93 & 6,80 & 5,66 & 0,65 \\
\hline 2. Independência e determinação & 48 & 2,00 & 7,00 & 5,90 & 0,84 \\
\hline $\begin{array}{l}\text { 3. Autoconfiança e capacidade de } \\
\text { adaptação a situações }\end{array}$ & 48 & 2,83 & 6,83 & 5,27 & 0,97 \\
\hline Escore total da Escala de Resiliência & 48 & 72 & 166 & 140,31 & 16,429 \\
\hline
\end{tabular}

Considerando que 7 é a pontuação máxima para cada fator de resiliência e a mínima é 1 , verificou-se que o fator mais bem pontuado pelas adolescentes foi “independência e determinação", com 95,83\% $(n=46)$ delas pontuando entre 5 e 7 , somente duas pontuaram abaixo de 5 , uma pontuou 4,75 e outra 2 .

O fator "Autoconfiança e capacidade de adaptação a situaçôes" apresentou a menor média entre os fatores da $\operatorname{ER}(5,27)$, no entanto, verifica-se que mais da metade das adolescentes, 64,5\% ( $\mathrm{n}=31)$, tiveram uma pontuação entre 5 a 6,83 , considerado alta. Já, 29,16\% $(\mathrm{n}=14)$ delas, pontuaram entre 4 e 4,83 e apenas $6,25 \%(\mathrm{n}=3)$ apresentaram uma pontuaçáo entre 2,83 e 3,33.

O fator "Resolução de ações e valores" da ER é composto por itens que transmitem a ideia de amizade, realização, satisfação e significado da vida. Este foi o fator com o segundo maior número de pontuaçóes altas, pois $89,58 \%(\mathrm{n}=43)$ das mães pontuaram entre 5 e 6,80 , já $8,33 \%(n=4)$ entre 4,40 e 4,80, e somente uma pontuou 2,93 .

\section{Caracterização do Apoio Social}

$\mathrm{Na}$ Escala de Apoio Social (MOS) aplicada neste estudo, a pontuação mínima é 2 e a máxima é 10 . Das adolescentes, 50\% ( $\mathrm{n}=24)$ obtiveram pontuaçóes muito altas, entre 9 e 10,29; 16\% $(\mathrm{n}=14)$ pontuaram entre 7,66 e 8,$88 ; 10,41 \%(n=5)$ entre 5,11 e 6,66, e apenas 10,41\% (n=5) entre 3,33 e 4,94 e a média obtida foi de 8,24. Verifica-se que 79,16\% das pesquisadas alcançaram acima de 7,66 pontos. Um número bem reduzido de participantes fez 4 pontos ou menos (Tabela 2).

Tabela 2. Caracterização do apoio social das mães adolescentes e das dimensões que compõem a Escala de Apoio Social

\begin{tabular}{|c|c|c|c|c|c|}
\hline Dimensões & n & $\begin{array}{c}\text { Mínimo } \\
\text { do } \\
\text { estudo }\end{array}$ & $\begin{array}{c}\text { Máximo } \\
\text { do } \\
\text { estudo }\end{array}$ & Média & $\begin{array}{l}\text { Desvio- } \\
\text { padrão }\end{array}$ \\
\hline 1. Afetiva & 48 & 1,33 & 5,00 & 4,19 & 0,97 \\
\hline 2. Material & 48 & 1,50 & 5,00 & 3,94 & 1,09 \\
\hline 3. Interação social positiva & 48 & 1,25 & 5,00 & 3,85 & 1,04 \\
\hline 4. Informação & 48 & 1,50 & 5,00 & 3,73 & 0,88 \\
\hline 5. Emocional & 48 & 1,25 & 5,00 & 3,59 & 1,14 \\
\hline Escore total da Escala de Apoio Social (MOS) & 48 & 3,33 & 10,00 & 8,24 & 1,89 \\
\hline
\end{tabular}

Observou-se que a maior pontuação das dimensôes da EAS (MOS) apresentada foi 5 e a menor foi 1 , a maior média apresentada foi na dimensão "Afetiva" $(4,19)$, seguida pelo "Material" $(3,94)$ e após, pela "Interação social positiva" $(3,85)$ e a menor média encontrada foi na dimensão "Emocional" $(3,59)$.

Das entrevistadas, 70,83\% ( $\mathrm{n}=34)$ obtiveram pontuaçóes entre 4 e 5 na dimensão "Afetiva”, que refere às suas percepções com relação a expressóes 
de afeto recebido que as façam se sentir queridas. $18,75 \%(\mathrm{n}=9)$ pontuaram entre 3 e 3,37 e $10,41 \%$ $(n=5)$ entre 1,33 e 2,67, sendo a dimensão melhor pontuada.

Apesar de mais da metade 66,66\% ( $n=32)$ das jovens apresentarem pontuações altas na dimensão "Material" da EAS (MOS), somente 10,41\% ( $\mathrm{n}=5)$ pontuaram entre 3 e 3,50 e uma porção considerável, $22,91 \%(n=11)$, tiveram pontuaçôes mais baixas, entre 1,50 e 2,75 .

A dimensão "Material" da EAS (MOS) se refere à percepção das adolescentes quanto à ajuda que recebem para realizar as tarefas diárias e para ir ao médico. Uma porcentagem expressiva pontuou baixo nesta dimensão, pois 22,91\% $(\mathrm{n}=11)$ delas atingiram entre 1,50 à 2,75 pontos e $10,41 \%(n=5)$ entre 3 à 3,50.

Em relação à dimensão "Interação social positiva” da EAS (MOS), que se refere à percepção das adolescentes sobre o quanto elas podem contar com as pessoas para fazer coisas agradáveis, encontramos mais da metade delas, 56,25\% $(n=27)$ que pontuaram entre 4 e 5; 20,83\% (n=10) entre 3 e 3,75 e $22,91 \%(n=11)$ entre 1,25 e 2,75 , podendo ser observado que uma porcentagem significativa obteve uma pontuação mais baixa para este fator.

Metade $(\mathrm{n}=24)$ das entrevistadas pontuaram entre 4 e 5 na dimensão "Informação", que se refere à percepção sobre o quanto elas podem contar com pessoas significativas para dar bons conselhos em uma situação de crise ou de como lidar com um problema, 33,33\% $(\mathrm{n}=16)$ pontuaram entre $3 \mathrm{e}$ 3,75 e $16,66 \%(n=8)$ entre 1,50 e 2,75 . Nesta dimensão, poucas tiveram pontuaçóes muito baixas, aparentando que boa parte delas recebe informaçóes de pessoas significativas em suas vidas.

A dimensão "Emocional” da EAS (MOS), que se refere à percepção das mães sobre o quanto elas podem contar com pessoas para compreendê-las, confiar e compartilhar seus problemas e preocupaçóes, foi a pior pontuada pelas adolescentes: $29,16 \%$ $(n=14)$ pontuaram entre 1,25 e 2,75 ; já $20,83 \%$ $(n=10)$ entre 3,25 e 3,75 e $50 \%(n=24)$ entre 4 e 5.

Observou-se uma correlação direta entre o fator "Resolução de açóes e valores" da ER e a dimensão "Interação social positiva" da EAS (MOS) apresen- tada pelas pesquisadas. Quanto maior a "Interação social positiva", maior a "Resolução de açóes e valores" (rho = 0,338; $\mathrm{p}=0,019)$.

Identificou-se uma correlação inversa entre o fator 2 "Independência e determinação" da ER e a dimensão "afetiva" da EAS (MOS). Esse resultado mostra que quanto maior o "Apoio afetivo" recebido pelas mães adolescentes, menor a "Independência e determinação" apresentada por elas ( $\mathrm{rho}=-0,288 ; \mathrm{p}$ $=0,047)$ (Figura 1).

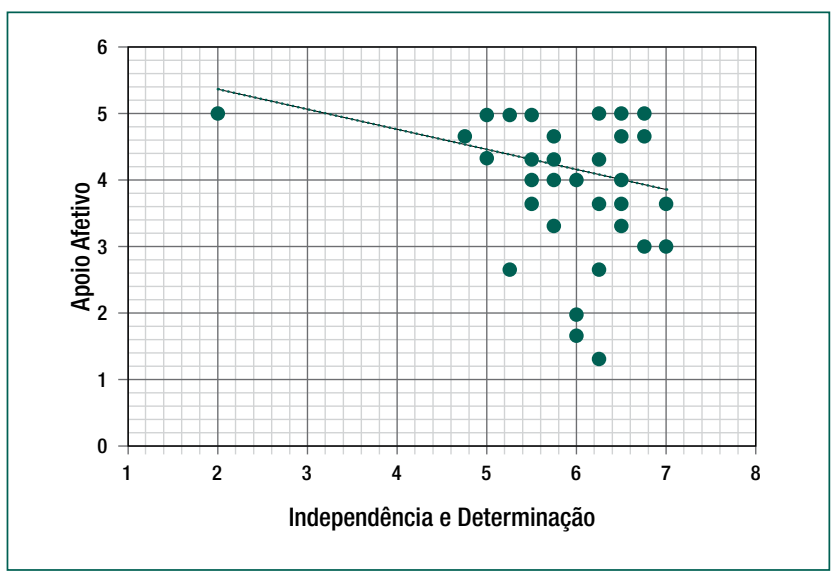

Figura 1. Correlação entre o fator 2, "Independência e determinação", da Escala de Resiliência e a dimensão "Afetiva" da Escala de Apoio Social (MOS)

\section{Discussão}

Estudos realizados em diversas regióes do Brasil corroboram a tendência da gestação/maternidade na adolescência ocorrer em maior número acima dos 15 anos. ${ }^{(2,24-26)}$

$\mathrm{O}$ atraso escolar significativo dentre as mães/gestantes adolescentes indica falta de apoio socioeconômico na gestação, está associada ao abandono escolar e consequentemente à dificuldade de retomar a educação formal para concluir o ensino fundamental ou médio, com impacto significativo para a vida destas adolescentes que se encontram em maior proporção ao final da adolescência 18 e 19 anos..$^{(2,8,25,27,28)}$

Sendo a resiliência um constructo dinâmico e construído por meio das experiências de vida de cada indivíduo, este estudo mostrou que a experiência materna na adolescência contribui para um comportamento mais resiliente destas mães. ${ }^{(10,12)}$ 
Alguns autores identificaram a maternidade como uma realização pessoal que revela a intenção consciente da adolescente de tornar-se máe, podendo representar um projeto de vida que a faz ser reconhecida como mulher e mãe. ${ }^{(26,28,29)}$ Porém, essas adolescentes continuam desejando estudar, trabalhar e investir na qualidade da relação mãe-filho, com a determinação de que ambos terão um futuro melhor. ${ }^{(26,28,30)}$

Entretanto, as mães adolescentes entrevistadas estão inseridas em um contexto de vulnerabilidade social. A escassez de políticas públicas efetivas no auxílio da continuidade dos estudos e a dificuldade ao acesso às creches impactam negativamente na inserção no mercado de trabalho e as transformam em um grupo com considerável grau de vulnerabilidade. ${ }^{(2,8,28,31)}$ Tais elementos acabam se configurando em um panorama de adversidade social da maternidade na adolescência.

Quanto mais as mães adolescentes pesquisadas tiveram relaçóes de afeto, menos independentes/determinadas elas se apresentaram e quanto mais inseridas numa união estável/casadas, menos continuaram estudando. Estes resultados podem refletir a forte aceitação de estereótipos acerca dos papéis tradicionais entre homens e mulheres que identificam a maternidade e a domesticidade como trajetórias naturais da mulher, ${ }^{(32)}$ fazendo com que abandonem os estudos e as possibilidades de qualificação profissional, prejudicando o seu potencial. ${ }^{(33)}$ No entanto, mais pesquisas são necessárias para compreender estas correlaçóes verificadas neste estudo.

Sendo assim, o apoio social no contexto de vida destas mães adolescentes, como o apoio familiar e do companheiro são imprescindíveis para dar suporte financeiro e ajudar nos cuidados diários da criança, para que possam vivenciar o cuidar de seu filho de forma mais tranquila. ${ }^{(28-30,34)}$

A família é considerada como importante fonte de apoio e de comunicação com as mães adolescentes e a sua falta pode ser prejudicial, sendo necessário que os profissionais da saúde sejam um canal aberto de informação, oferecendo-lhes subsídios para que desenvolvam habilidades e competências para tomar decisóes mais conscientes. ${ }^{(30)}$

Considerando os achados, na dimensão material da EAS (MOS), é essencial incluir nos servi- ços de saúde em conjunto com a educação, espaços onde os adolescentes possam discutir e refletir sobre projetos de vida, desejos e expectativas quanto ao futuro, bem como sobre os papéis femininos e masculinos na parentalidade esperados na sociedade e no contexto em que estão inseridos. Tais espaços podem apoiar o processo emancipatório e de empoderamento que contribua para torná-los mais sensíveis a adotarem posturas de corresponsabilização das tarefas e igualdade de gênero, sobretudo no cuidado das crianças pequenas. ${ }^{(34)}$

De modo geral, as mães deste estudo apresentaram atributos como independência, determinação, autoconfiança, capacidade de adaptação a situaçóes, realização pessoal, satisfação e significado de vida, caracterizando-as como resilientes em meio ao contexto de vida em vulnerabilidade. No entanto, é importante observar a necessidade de oferecer atenção especial ao fortalecimento dos atributos de autoconfiança e capacidade de se adaptar às situaçóes em virtude da menor pontuação neste fator, em relação aos outros fatores da ER.

Há espaço para mais pesquisas que desvelem os fatores que fortalecem ou não o apoio que elas recebem, especialmente na dimensão emocional da EAS (MOS), uma vez que neste estudo, muitas delas se sentiam pouco compreendidas, não conseguindo compartilhar e confiar suas preocupaçóes às pessoas que as cercam. Estes elementos se constituíram nas limitaçóes do presente estudo.

\section{Conclusão}

Observou-se nesse estudo que o apoio afetivo refletiu na diminuição da independência e da determinação, o que pode ter prejudicado o potencial de resiliência das mães adolescentes no enfrentamento das adversidades durante o período da gestação e maternidade. As mães que se sentiam seguras emocionalmente apresentaram maior resiliência nas situaçóes adversas. A evasão escolar caracteriza-se como achado primordial para entender que a maternidade na adolescência afeta diretamente a construção dos projetos de vida das mães adolescentes o que impacta diretamente na perpetuação do ciclo da pobreza e miséria. 


\section{Agradecimentos}

À Coordenação de Aperfeiçoamento de Pessoal de Nível Superior (CAPES) pela concessão de bolsa de mestrado para Bianca Gansauskas de Andrade e auxílio financeiro para a realização desta pesquisa.

\section{Colaborações}

Andrade BG, Assis CA, Lima DCM, Neves LF, Silva LA, Silva RC, Fracolli LA e Chiesa AM contribuíram com a concepção do estudo, análise e interpretação dos dados, revisão do artigo, revisão crítica do conteúdo intelectual e aprovaçáo da versáo final a ser publicada.

\section{Referências}

1. Fundo das Nações Unidas para a Infância (UNICEF). Situação da Adolescência Brasileira 2011. 0 direito de ser adolescente: oportunidade para reduzir vulnerabilidades e superar desigualdades. Brasilia (DF): UNICEF; 20211 [citado 2019 Jun 20]. Disponível em: https://www.tjdft.jus.br/informacoes/infancia-e-juventude/ publicacoes-textos-e-artigos/publicacoes/publicacoes-1/situacao-daadolescencia-brasileira-2011

2. Nascimento MS, Lippi UG, Santos AS. Social and individual vulnerability and teenage pregnancy. Rev Enferm Atenção Saúde. 2018;7(1):15-29.

3. Brasil. Ministério da Saúde. Secretaria de Atenção à Saúde. Departamento de Ações Programáticas Estratégicas. Marco teórico e referencial: saúde sexual e saúde reprodutiva de adolescentes e jovens. Brasília (DF): Ministério da Saúde; 2007. 56 p. (Série B. Textos Básicos de Saúde). [citado 2015 Nov 4]. Disponível em: http://bvsms. saude.gov.br/bvs/publicacoes/07_0471_M.pdf

4. Ayres JR, Paiva V, França Jl. From natural history of disease to vulnerability. In: Parker R, Sommer M. Routledge handbook in global public health. New York: Routledge; 2011. p. 98-107.

5. Silva DI, Chiesa AM, Verissimo ML, Mazza VA. Vulnerability of children in adverse situations to their development: proposed analytical matrix. Rev Esc Enferm USP. 2013;47(6):1397-402.

6. Alegria FV, Schor N, Siqueira AA. Gravidez na adolescência: estudo comparativo. Rev Saúde Pública. 1989;23(6):473-77.

7. Lima LS, Tocci HA. Gravidez na adolescência: intercorrências e prematuridade. Rev Enferm UNISA. 2001;2:62-7.

8. Novellino MS. Um estudo sobre as mães adolescentes brasileiras. Physis. 2011;21(1):299-318.

9. Fundo de População das Nações Unidas (UNFPA). Maternidade precoce: enfrentando o desafio da gravidez na adolescência. Boa Vista: UNFPA; 2013 [citado 2019 Jun 20]. Disponível em: http://www.unfpa. org.br/Arquivos/swop2013.pdf
10. Hoga LA. Adolescent maternity in a low income community: experiences revealed by Oral history. Rev Lat Am Enfermagem. 2008;16(2):280-6.

11. Duncan S. What's the problem with teenage parents? And what's the problem with policy? Critical Social Policy. 2007;27(3):307.

12. Ribeiro PM, Gualda DM. Gestação na adolescência: a construção do processo saúde-resiliência. Esc Anna Nery. 2011;15(2):361-71.

13. Silva KS, Rozenberg R, Bonan C, Chuva VC, Costa SF, Gomes MA. Gravidez recorrente na adolescência e vulnerabilidade social no Rio de Janeiro (RJ, Brasil): uma análise de dados do Sistema de Nascidos Vivos. Cien Saude Colet. 2011;16(5):2485-93.

14. Due P, Holstein B, Lund R, Modvig J, Avlund K. Social relations: network, support and relational strain. Soc Sci Med. 1999;48(5):661-73.

15. Sherbourne CD, Stewart AL. The MOS social support survey. Soc Sci Med. 1991;32(6):705-14.

16. Griep RH, Chor D, Faerstein E, Werneck GL, Cláudia S, Lopes CS. Validade de constructo de escala de apoio social do Medical Outcomes Study adaptada para o português no Estudo Pró-Saúde. Cad Saúde Pública. 2005;21(3):703-14.

17. Valla W. Educação popular, saúde comunitária e apoio social numa conjuntura de globalização. Cad Saúde Pública. 1999;15(Suppl 2):S7-S14.

18. Cassel J. An epidemiological perspective of psychosocial factors in disease etiology. Am J Public Health. 1974;64(11):1040-43.

19. Antunes C. Resiliência: a construção de uma nova pedagogia para uma escola pública de qualidade. Rio de Janeiro: Vozes; 2003.

20. Faul F, Erdfelder E, Lang AG, Buchner A. G*Power 3: A flexible statistical power analysis program for the social, behavioral, and biomedical sciences. Behav Res Methods. 2007;39(2):175-91.

21. Pesce RP, Assis SG, Avanci JQ, Santos NC, Malaquias JV, Carvalhaes R. Adaptação transcultural, confiabilidade e validade da escala de resiliência. Cad Saúde Pública. 2005;21(2):436-48.

22. Wagnild GM, Young HM. Development and psychometric evaluation of resilience scale. J Nurs Meas. 1993;1(2):165-78.

23. Brasil. Ministério da Saúde. Conselho Nacional de Saúde. Resolução n 466 , de 12 de dezembro de 2012. Aprova as diretrizes e normas regulamentadoras de pesquisas envolvendo seres humanos. Brasília (DF): Ministério da Saúde; 2013 [citado 2019 Jun 20]. Disponível em: https://bvsms.saude.gov.br/bvs/ saudelegis/cns/2013/res0466_12_12_2012.html

24. Instituto Brasileiro de Geografia e Estatística (IBGE). Diretoria de Pesquisas, Coordenação de População e Indicadores Sociais. Estimativas da população residentes para os municípios e para as unidades da Federação Brasileira com data de referência em $1^{\circ}$ de julho de 2015. Rio de Janeiro: IBGE; 2015 [citado 2019 Jun 20]. Disponível em: https:// biblioteca.ibge.gov.br/visualizacao/livros/liv97746.pdf

25. Fernandes MM, Santos AG, Esteves MD, Vieira JS, Neto BP. Fatores de riscos associados à gravidez na adolescência. Rev Enferm UFPI. 2017;6(3):53-8.

26. Lima FB, Lira GG, Melo RA, Mola R, Fernandes FE. Maternidade: significados atribuídos por adolescentes primíparas. Rev Enferm UFPE Online. 2017;11(3):1163-70.

27. Oliveira-Campos M, Nunes ML, Madeira FC, Santos MG, Bregmann SR, Malta DC, et al. Sexual behavior among Brazilian adolescents, National Adolescent School-based Health Survey (PeNSE 2012). Rev Bras Epidemiol. 2014;17(4 Suppl 1):S116-30.

28. Lima TN, Coviello DM, Lima MN, Alves ES, Davim RM, Bousquat AE. Social support networks for adolescent mothers. Rev Enferm UFPE Online. 2016;(10 Suppl 6):4741-50. 
29. Andrade PR, Ribeiro CA, Ohara CV. Maternidade na adolescência: sonho realizado e expectativas quanto ao futuro. Rev Gaúcha Enferm. 2009;30(4):662-8.

30. Bordignon SS, Jacondino MB, Meincke SM, Soares MC. Aspectos educacionais e a parentalidade na adolescência. Rev Pesq Cuidado Fundam Online. 2013;5(1):3285-92.

31. Monterrosa-Castro A, Ulloque-Camaño L, Arteta-Acosta C. Estrategias para mejorar el nivel de resiliencia en adolescentes embarazadas. Investig Enferm Imagen Desarrollo. 2018;20(2):1-12.
32. Chacham AS, Maia MB, Camargo MB. Autonomia, gênero e gravidez na adolescência: uma análise comparativa da experiência de adolescentes e mulheres jovens provenientes de camadas médias e populares em Belo Horizonte. Rev Bras Estud Popul. 2012;29(2):389-40.

33. Sousa MC, Gomes KR. Conhecimento objetivo e percebido sobre contraceptivos hormonais orais entre adolescentes com antecedentes gestacionais. Cad Saúde Pública. 2009,25(3):645-54.

34. Zanettini A, Souza JB, Aguiar DM. The interfaces of adolescent and adult mothers first experience. Rev Enferm Centro Oeste Mineiro. 2017;7:e1987. 